\title{
3 Dimensional Wire Array for Connection between Multi Layers
}

\author{
Member Satoshi Konishi * (IIS, University of Tokyo), \\ Non-Member Kouji Matsushita (Fuji Electric Co., Ltd.), \\ Non-Member Tomoaki Goto (Fuji Electric Co., Ltd.), \\ Member Hiroyuki Fujita. (IIS, University of Tokyo)
}

\begin{abstract}
Summary
We have proposed the concept of distributed micro motion systems (DMMS) composed of micro cell array. We focused on the multi-layered fabrication process to integrate such micro devices as actuators, sensors and circuits for implementation of a micro cell array. In this case, each devices are produced on a separate substrate and connected together after fabrication. This paper describes the 3 dimensional (3-D) wire array which have been developed for the interconnection between these substrates.
\end{abstract}

Key words: 3-D wire array, Distributed Micro Motion Ssystems, micro cell array, multi-layered fabrication, plasma etching

\section{Introduction}

We have proposed the concept of distributed micro motion systems (DMMS) in order to make the best use of the advantage and overcome the limitation of MEMS(1). The key idea is to coordinate simple motions of many actuators arranged on a wafer in order to perform a more complicated task. In considering the control of DMMS, however, the amount of control signals becomes huge and wiring to each cell from outside must be impractical. The integration of actuators, sensors and circuits by IC-compatible micromachining makes it possible to compose an inteligent micro cell with a primary servomechanism. This leads to the concept of autonomous distributed micromachines $(A D M)(2)$ composed of many micro cells which are smart enough to decide its behavior by itself.

Several approaches have been proposed to integrate such $\mathrm{mi}$ cro devices as actuators, sensors and circuits. Integration of micro devices on the same substrate requires redesign of either MEMS or IC process (3). That is to say, MEMS process uses high temperature steps so as not to be allowed after IC process. We focused on the multi-layered fabrication process in which each device array is supplied on a separate substrate respectively as shown in Fig. 1. In this case, we can use MEMS process without limitation caused by IC process and can also utilize IC foundries to fabricate IC substrate. MEMS substrate should be exposed to the external environments and interact with each other. On the other hand, IC substrate used to be protected by the passivation layer as the packaging. The multi-layered fabrication will satisfy both requirements of MEMS and IC substrates. We can name the developed field emitters with thin film transistors(4) as one of examples using this technology.

\footnotetext{
*Present affilation : Ritsumeikan University
}

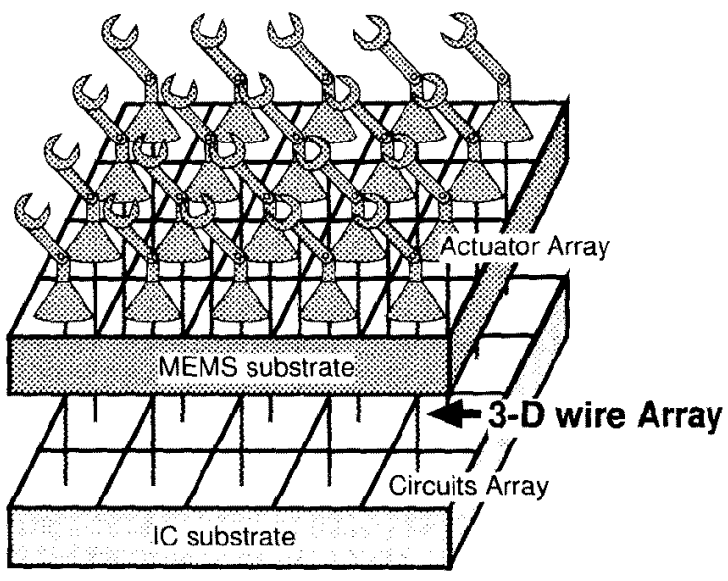

Fig. 1. Schematic drawing of the multi-layer structure

This paper keeps in mind with implementation of microactuator $\operatorname{array}(1)$ for an in-plane positioner. The developed microac tuator array is driven electrostatically.

Actuator and controller of each micro cell is fabricated on separate substrates in using the multi-layered fabrication process. Interconnection method between multi layers becomes necessary. DMMS composed of a micro cell array require individual connections between actuators and controllers. Some trials have been reported on the interconnection technology through wafers(5), however, the density of wires is still limited. This paper describes the arrayed 3 dimensional (3-D) wires which have been developed for the interconnection between multi layers.

\section{3-D wire array}

The 3-D wire array is fabricated into the actuator substrate which is to be bonded and connected with the IC substrate. We 
(a)

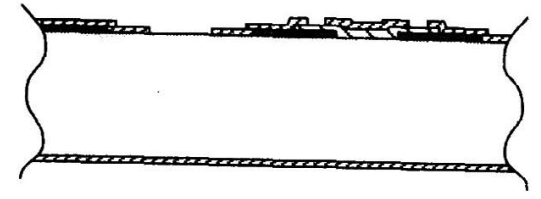

(b)

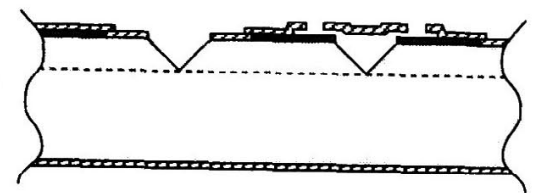

(c)

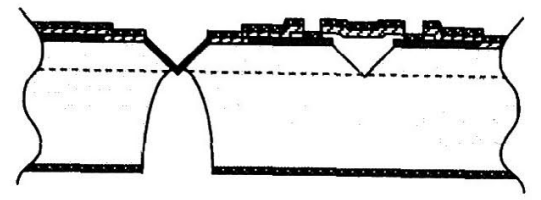

(d)

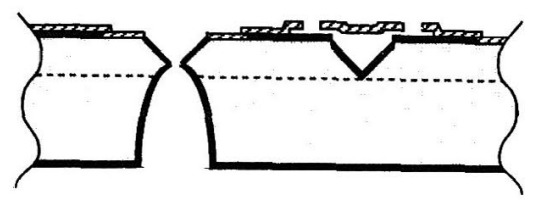

(†)

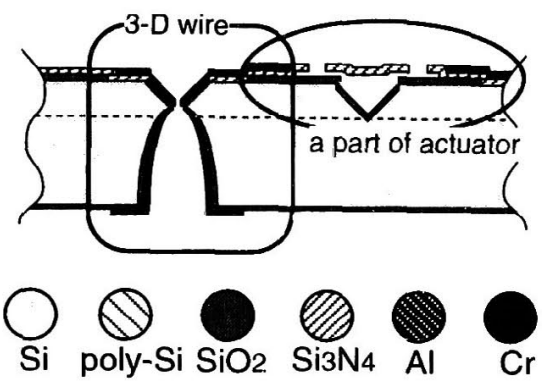

Fig. 2. Fabrication process.

have previously reported 3-D wire array using the anisotropical conductive polymer for bonding(6). Dry etching with mixture of gas $\left(\mathrm{SF}_{6}, \mathrm{CHF}_{3}\right.$ and $\left.\mathrm{O}_{2}\right)$ from both sides of substrate was used to obtain deep through holes. Furthermore, metal electroplating method was employed to form wires and bumps on the holes. We used $\mathrm{Ni}$ as a material of wire. A fabricated hole was 150 $\mu m$ in diameter at the entrance and $300 \mu m$ in depth. Height of the bump was $7 \mu \mathrm{m}$. Average of resistance between top and backside was about $20 \Omega$ and insulation between neighbor wires was confirmed completely. Arrayed 64 wires were fabricated in $1.5 \times 6 \mathrm{~mm}^{2}$ area and fabrication yield was more than $90 \%$.

This paper presents the 3-D wire array for integration with microactuator array. As metioned above, the developed microactuator array for the positioner is driven by electrostatical force between opposing electrodes. Most of structers for microactuators are fabricated after fabrication process of 3-D wire array. We have improved 3-D wire array to solve the following problems: (1) Roughness on the surface of substrate; (2) Density of the wire array. Roughness caused by holes of 3-D wire array brings fatal influences on the following fabrication process of 3-D wire array.

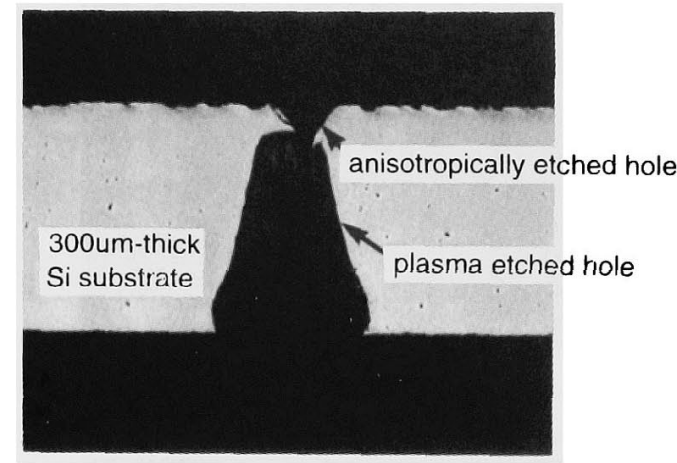

Fig. 3. Cross-sectional view of fabricated through hole

High density of 3-D wire array is required to increase density o the micro cell array.

\section{Fabrication of 3-D wire array}

Combination of the dry etching and the anisotropical we etching of $\mathrm{Si}$ is employed in order to minimize The surface rough ness after fabrication process of 3-D wire array. The etching witl $\mathrm{KOH}$ is applied from topside of the substrate and the plasm: etching is carried out from backside of the same substrate. Thi: plasma etching has been developed to obtain deep holes wit1 vertical sidewalls $(7)$.

Process sequence is as follows (see Fig. 2):

(1) A (100) Si wafer is thermally oxidised and patterned in the top thermal $\mathrm{SiO}_{2}$ using photo resist mask in order to form mask for etching of upper hole array (Fig. 2(a)).

(2) Taperd holes are etched from top side of $300 \mu \mathrm{m}$ thicl substrate by anisotropical wet etching of (100) Si. Each hole is 51 $\mu \mathrm{m}^{2}$ at its entrance and $40 \mu \mathrm{m}$ in depth. After etching, therma $\mathrm{SiO}_{2}$ is removed completely(Fig. 2(b)).

(3) $1 \mu \mathrm{m}$ thick Al layer is evaporated on both surface of the wafer as an etching mask. After alignment between patterns fol the backside holes and fabricated upper hole array, $\mathrm{Al}$ mask i: patterned by wet etching. Aperture of a mask-pattern for a holk is $50 \mu \mathrm{m}$ in diameter.

(4) The plasma etching process with $\mathrm{SF}_{6} / \mathrm{O}_{2}$ gas mixture i: applied to etch deep holes from backside until through holes art obtained. This etching also progress in lateral direction as a socalled undercut etching. As a result of this plasma etching fron backside, each hole becomes about $200 \mu \mathrm{m}$ in diameter at it: entrance and more than $260 \mu \mathrm{m}$ in depth (Fig. 2(c)).

(5) After removing $\mathrm{Al}$ mask, the wafer is thermally oxidisec again to isolate wires each other(Fig. 2(d)).

(6) Rather thick metal is sputterd from both sides of the wafer. The reason why we employ sputtering method here is that the step-coverage by deposition using sputtering is bette 


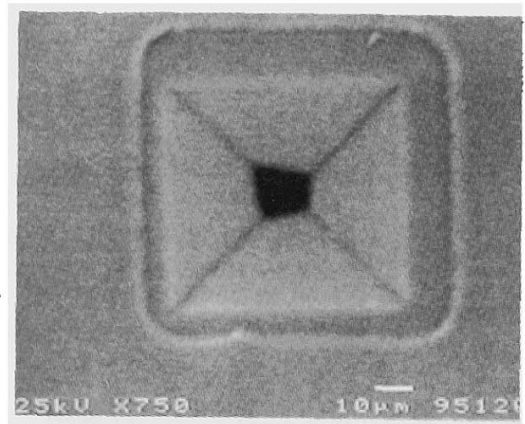

(a)

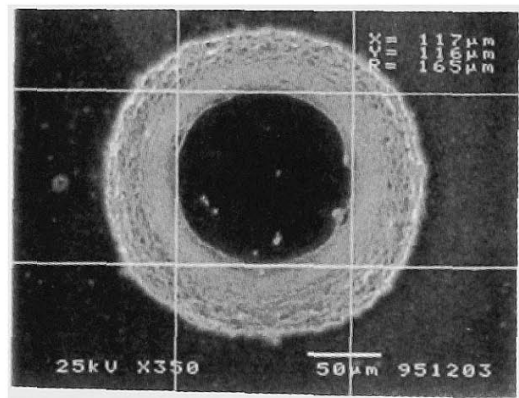

(b)

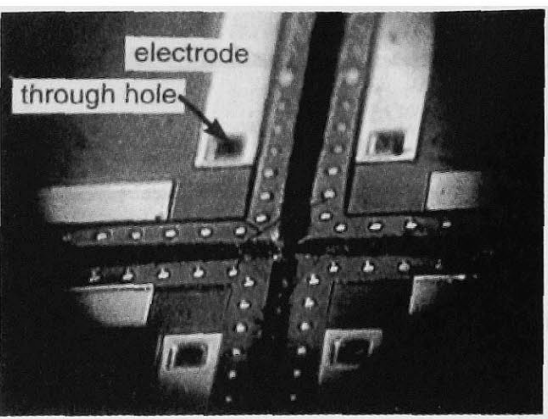

(a)

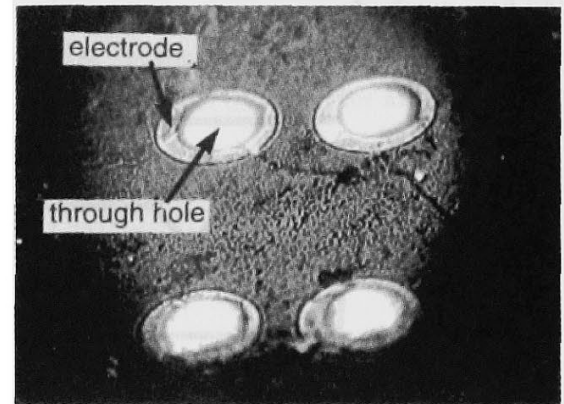

(b)
Fig. 4. Fabrication results of a through hole.(a)Top view, (b)Backside view.

than evaporation. Such metal as $\mathrm{Ni}$ and $\mathrm{Cr}$ is used for material of wires.

(7) Metal is patterned so as to form wires which is isorated each other(Fig. 2(e)). Electroplating is also used if it is necessary to form bump

(8) Fabrication of microactuators will be applied after these wiring process. In fact, we can design microactuators process progressing in parallel with the wiring process.

\section{Results}

Figure 3 shows a cross-sectional view of a fabricated through hole. We can see two kinds of tapered hole from both sides of wafer. The diameter where these two holes meet each other is about $30 \mu \mathrm{m}$. Top and backside views of the hole are shown in Fig. 4(a) and (b) respectively. Figure 5(a) and (b) show results of wiring on these holes. $1 \mu \mathrm{m}$ thick sputtered $\mathrm{Cr}$ is used as a material of wire. 3-D wire array composed of 144 wires has been fabricated in $8 \mathrm{~mm}^{2}$ area and average of resistance between top and backside was about $20 \Omega$. Each micro cell for positioner requires 4 wires and $1443-\mathrm{D}$ wires are for a $6 \times 6$ micro cell array. Insulation between neighbor wires is also completely confirmed. Microactuator structure, which works as an air-nozzle, is also fabricated beside the $3-\mathrm{D}$ wire as shown in Fig. 6. A part of structure of the micro actuator is fabricated in advance at the
Fig. 5. Results of wiring on the through hole.(a)Top view,(b)Backside view.

same time that the through hole wire is formed (see Fig. 2). After high temperature process (especially thermal dioxidation), remaining process is applied to complete structure of air-nozzles. Air-nozzles are made of polymide. Electrodes are also made in air-nozzles in order to drive nozzles electrostatically.

\section{Discussions}

We have developped the 3 -D wire array for the multi-layered method as shown in Fig.1. The anisotropical wet etching of (100) Si wafer is often used to obtain a through hole for a 3-D wire. Linder et al.(8) has developped technology to print patterns into anisotropically etched hole. Their technology allows us to form

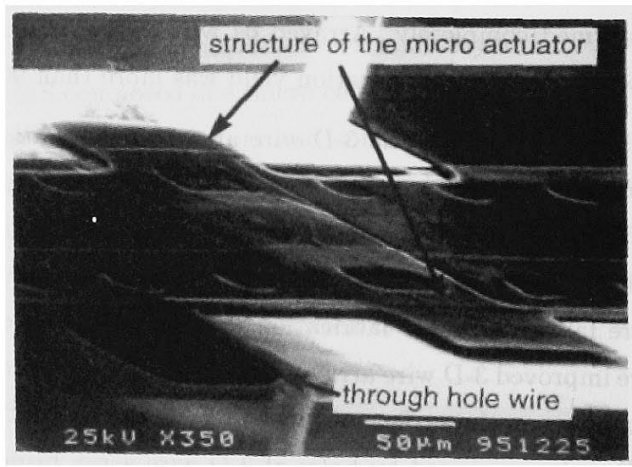

Fig. 6. Microactuator fabricated beside 3-D wire 


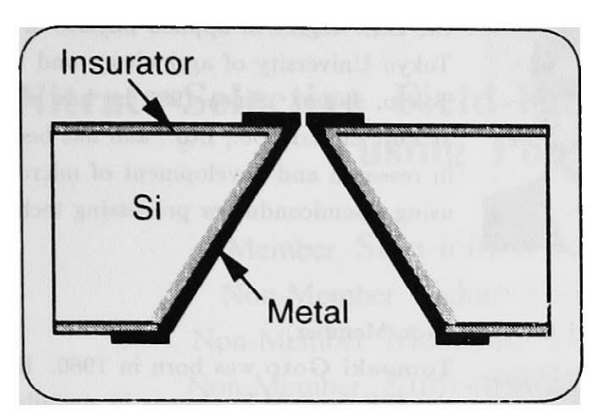

(a)

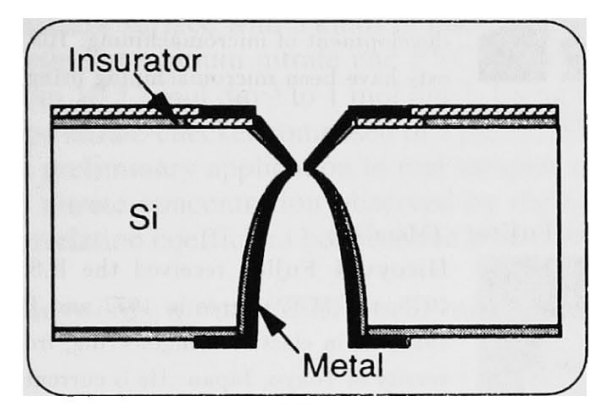

(b)

Fig. 7. Two types of through hole wire into $\mathrm{Si}$ wafer(cross-sectional view)

several wire patterns in a single hole. On the other hand, we employed approach using dry etching technology because of the following reasons:

(1)Etching of deep through holes with narrow apertures.

(2)Improvement of wire density.

(3)Reduction of surface area for wiring.

Recently much effort has been made in deep etching of $\mathrm{Si}(9)$. We employed plasma etching to obtain such deep holes in stead of RIE. This process is developed as 3-D microfabrication as mentioned before. It took about 4 hours to etch the $260 \mu \mathrm{m}$ deep holes by plasma etching though etching by RIE used to take more than 10 hours. The anisotropical wet etching of (100) Si wafer, however, is empoloyed to reduce roughness on the surface of wafer. That is why, high aspect ratio etching becomes all the more necessary to increase the density of the wire array. Let me compare properties between the technorogy using the anisotropically etched hole (see Fig. 7(a)) and our method (see Fig. 7(b)). The maximum number of through hole wires per unit area is given by

$$
n_{T H W} \simeq \frac{9}{4 \cdot\left(t_{\text {wafer }}+3 \mathrm{~g} / 2\right)^{2}} \quad\left[\mathrm{~cm}^{-2}\right]
$$

where $t_{\text {wafer }}$ is thickness of the wafer and $\mathrm{g}$ is gap between adjacent wires. While, the maximum density of through-holes by anisotropical wet etching is given by(8)

$$
n_{P I T} \simeq \frac{1}{2 \cdot t_{\text {wafeT }}^{2}} \quad\left[\mathrm{~cm}^{-2}\right]
$$

Therefore, for a $500 \mu \mathrm{m}$ thick wafer, the maximum number of through hole wires per unit area is calculated as shown in Table 1. Our result corresponds to the density of four wires patterned in a single pyramid-like pit. Furthermore, our method can provide stiffer structure than that using a big hole from the mechanical point of view.

Table 1. The maximum $\mathrm{n}$ per unit area

\begin{tabular}{|l||cc|c|}
\hline & $\mathrm{n}_{T H W}$ & $\mathrm{n}_{P I T}$ \\
\hline \hline Calculated value & $800 \quad($ at $\mathrm{g}=20 \mu m)$ & 200 \\
\hline
\end{tabular}

Surface area for the wire significantly contributes to the capacitance of the electroplated through hole wire. The capacitance of the plated through hole is given by $(8)$

$$
C=\epsilon_{o x} \frac{A_{\text {metal }}}{t_{o x}}
$$

where $\epsilon_{o x}$ is the permittivity of the silicon dioxide film, $A_{\text {metal }}$ is the total area of the metal and $t_{o x}$ is the thickness of the $\mathrm{SiO}_{2}$ layer. Table 2 shows the calculated capacitance, for a $300 \mu \mathrm{m}$ thick wafer, derived from each surface area of the covered metal respectively. The capacitance of wire $\mathrm{C}_{T H W}$ is less than the half of $\mathrm{C}_{P I T}(8)$. Generally speaking, calculated capacitance is not so low enough to transmit signals at high frequence. It becomes necessary to choose method to proper use.

Table 2. The capacitance of each hole

$$
\text { (at } t_{\text {wafer }}=300 \mu \mathrm{m} \text { ) }
$$

\begin{tabular}{|l||c|c|}
\hline & $\mathrm{C}_{T H W}$ & $\mathrm{C}_{P I T}$ \\
\hline \hline Calculated value & $4[\mathrm{pF}]$ & $9[\mathrm{pF}]$ \\
\hline
\end{tabular}

As mentioned above, most of microactuator strucures are fabricated after wiring process. The presented method, however, still has a limitation in temperture and requires modification of process. Replacement of wire material with more refractory metals, such as tungsten, is one of the solutions. In the presented method, such high temperature process as deposition of silicon nitride was applied in parallel with the process of wiring before treating metals. We don't have to redesign fabrication process 
if it is possible to finish high temperature process before using metals.

\section{Conclusion}

We have improved 3-D wire array which was developed for the interconnection between multi layers. We can see that the presented technology using the deep plasma etching supply 3-D wire structure with practical properties. We may reasonably conclude that the developed 3-D wire array will contibute to multilayer method for integration and packaging of micro devices.

\section{References}

(1) S. Konishi and H. Fujita: "A Conveyance System using Air Flow based on the concept of Distributed Micro Motion Systems", Journal of Microelectomechanical Systems, Vol.3, No.2, pp. 54-58 (June 1994)

(2) S. Konishi and H. Fujita: "System Design for Cooperative Control of a Microactuator Array", The IEEE Transactions on Industrial Electronics, Vol.42, No.5, pp. 449-454 (October 1995)

(3) K. Shaw and N. MacDonald: "Integrating SCREAM Micromachined Devices with Integrated Circuits", Proc. of Micro Electro Mechanical Systems, San Diego, California, USA, pp. 44-48 (February 11-15 1996)

(4) G. Hashiguchi, H. Mimura and H. Fujita: "Multi-layered Fabrication of Microstructure and Thin Film Transistors - Apprication to Polycrystalline Silicon Field Emitters Controlled by TFTs", Proc. of Micro Electro Mechanical Systems, San Diego, California, USA, pp. 49-53 (February 11-15 1996)

(5) T. Suzuki: "Electronics Magaine" in Japanese, Ohm press, pp. 44-45 (1994)

(6) S. Konishi, H. Fujita: "3 Dimensional Through Hole Wireing by Micromachining" in Japanese, The Transactions of the IEEJ (April 1996)

(7) T. Goto, K. Matsushita, K. Hirano: "Three Dimensional Microfabrication of Single-Crystal Silicon by Plasma Etching", IEICE Trans. Electron, vol. E78-C, No.2, pp.167-173 ( February 1995)

(8) S. Linder, H. Baltes, F. Gnaedinger and E. Doering: "Photolithography in Anisotropically Etched Grooves", Proc. of Micro Electro Mechanical Systems, San Diego, California, USA, pp. 38 43 (February 11-15 1996)

(9) H. Jansen, M. Boer, B. Otter and M. Elwnspoek: "The Black Silicon Method IV", Proc. of Micro Electro Mechanical Systems, Amsterdam, the Netherland, pp. 88-93 (January 29-February 2 1995)

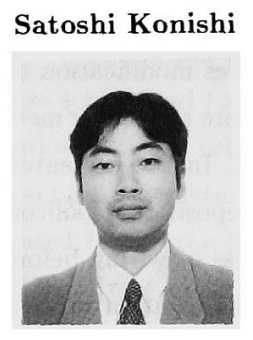

(Member)

Satoshi Konishi received the B.S. degree in 1991 in electronics engineering, the M.S. degree in 1993 and $\mathrm{Ph} . \mathrm{D}$ degree in 1996 in electrical engineering, all from the University of Tokyo, Japan. In 1996, he joined Ritsumeikan University, as a lecture. He is investigating microelectromechanical systems and especially interested in distributed micro motion systems.

\section{Kouji Matsushita (Non-Member)}

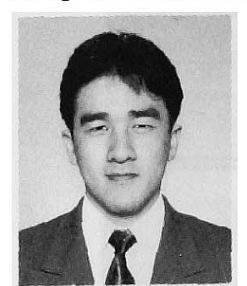

Tomoaki Goto was born in 1968. He received the B.E. degree in applied physics in 1990 from Tokyo University of agriculture and technology, Tokyo, Japan. Since 1990 he has been worked at Fuji Electric Co., Ltd., and has been engaged in research and development of micromachining using a semiconductor processing techinque

\section{Tomoaki Goto (Non-Member)}

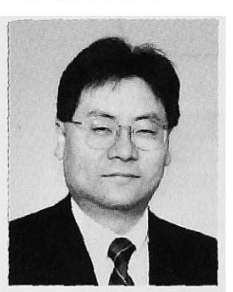

Tomoaki Goto was born in 1960. He received the B.E. and M.E. degree in metallurgical engineering from Yokohama National University, Kanagawa, Japan in 1983 and 1985, respectively. Since 1985 he has been worked at Fuji Electric Co., Ltd., and has been engaged in research and development of micromachining. His main interests have been micromachining using a semicon-

ductor processing

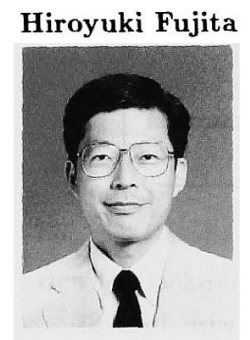

(Member)

Hiroyuki Fujita received the B.S. degree in 1975, the M.S. degree in 1977 and Ph.D degree 1980, all in electrical engineering, from the University of Tokyo, Japan. He is currently a Professor in the Institute of Industrial Science, University of Tokyo, where he joined the fuculty in 1980 . His current interests are micromachining technology, microactuators, and micromechatoronics.

(平成 8 年 3 月 29 日受付、同 8 年 11 月 1 日再受付) 